\title{
Magnetic jam in the corona of the Sun
}

\author{
F. Chen ${ }^{1}$, H. Peter ${ }^{1 \star}$, S. Bingert ${ }^{2}$ and M. C. M. Cheung ${ }^{3}$
}

The outer solar atmosphere, the corona, contains plasma at temperatures of more than a million kelvin-more than 100 times hotter than the solar surface ${ }^{1}$. How this gas is heated is a fundamental question tightly interwoven with the structure of the magnetic field ${ }^{2}$. Together this governs the evolution of coronal loops, the basic building block prominently seen in X-rays and extreme ultraviolet (EUV) images ${ }^{3}$. Here we present numerical experiments accounting for both the evolving three-dimensional structure of the magnetic field and its complex interaction with the plasma. Although the magnetic field continuously expands as new magnetic flux emerges through the solar surface, plasma on successive field lines is heated in succession, giving the illusion that an EUV loop remains roughly at the same place. For each snapshot the EUV images outline the magnetic field. However, in contrast to the traditional view, the temporal evolution of the magnetic field and the EUV loops can be quite different. This indicates that the thermal and the magnetic evolution in the outer atmosphere of a cool star should be treated together, and should not be simply separated as predominantly done so far.

The dominance of the magnetic field in the corona gives rise to the sharp appearance of coronal loops seen in EUV or X-ray images (see Fig. 1): if energy is deposited on a magnetic field line, heat conduction in the ionized gas will redistribute that energy efficiently along only that field line (but not across). Consequently, the plasma along that field line becomes visible in EUV and X-ray images: the coronal emission shows the magnetic field in a way similar to that in which iron filings are used in school to show the field lines of a magnet.

Because direct measurements of the coronal magnetic field are notoriously difficult ${ }^{4}$, mainly extrapolations of the observed magnetic field at the surface provide the magnetic information in the corona ${ }^{5}$. Stereoscopic observations can provide the threedimensional (3D) structure of coronal loops ${ }^{6}$. Comparing EUV images and extrapolations reveals that loops seen in EUV images indeed outline field lines ${ }^{7}$. This paradigm underlies both onedimensional modelling ${ }^{2,8,9}$, where the thermodynamics of the coronal plasma is often treated in detail along assumed static field lines, and magnetofrictional modelling ${ }^{10,11}$, where an instantaneous thermal equilibrium is often assumed along dynamic field lines. On the real Sun we will not find these extreme cases, but a changing magnetic field hosting plasma with an evolving thermal structure, as described by the full equations of magnetohydrodynamics (MHD; ref. 2).

Models accounting for this 3D structure and evolution of the solar corona point to a mismatch between magnetic and thermal structure $^{12}$, which plays an important role in understanding the cross-section of coronal loops ${ }^{13}$. The thermal evolution-that is, when plasma gets heated and when a loop becomes visible in EUV images-is coupled in a much more subtle time-dependent way to field lines and heat input than often assumed. Thus, in general the appearance of coronal loops depends not just on the instantaneous position and shape of field lines but also on their evolution.

We show that such scenarios are realistic for situations on the Sun; thus, our understanding of the structure and evolution of the solar corona, and ultimately the heating processes, will have to fully acknowledge the intimate interaction of the thermal evolution of coronal loops and the changing magnetic structure. In a similar way, the thermal evolution of coronal loops (in flares) has long been recognized to be caused by an interaction with a time-dependent magnetic structure $e^{2,14}$.

To investigate the corona above a solar active region we conduct a $3 \mathrm{D}$ numerical experiment. For this we solve the problem of MHD in which the induction equation describing the magnetic field is coupled to the conservation of mass, momentum and energy of the plasma. In the energy balance we account for heat conduction along the magnetic field, optically thin radiative losses and heating through Ohmic dissipation. Our model follows the philosophy of previous studies where the magnetic field is driven at the surface of the Sun, which is the lower boundary of the model ${ }^{15-17}$. In contrast to earlier models, we drive our system by a separate model of an emerging sunspot pair ${ }^{18}$. This way coronal loops form in the emerging active region in response to the enhanced Poynting flux into the corona at locations where magnetic field is pushed around, similar to flux braiding ${ }^{19}$ or flux-tube tectonics ${ }^{20}$. This new study on the evolution of thermal and magnetic properties is based on the same simulation as used before to investigate the formation of active region loops ${ }^{21}$. Further details of these 3D models and their success in describing coronal observations is discussed in Supplementary Section 1. The comparison in Fig. 1 illustrates that 3D models capture essential observational signatures, which is a significant step forward in understanding the structure, dynamics and heating of the corona, one of the enigmatic problems in astrophysics.

To study the relation of the magnetic field to coronal loops seen in emission we have to follow the temporal evolution of both in the simulation. The procedure to follow (a bundle of) magnetic field lines as well as an EUV loop is described in Supplementary Section 2. From the output of the MHD simulation, we use the temperature and density at each grid point to evaluate the coronal emission. Integrating along a line-of-sight we then obtain synthetic observations that can be treated as real ones ${ }^{22}$. Here we synthesize the coronal emission as it would be seen by the atmospheric imaging assembly (AIA; ref. 23) onboard NASA's Solar Dynamics Observatory. For our analysis we concentrate on the $193 \AA$ filter, which is dominated by emission from Fe XII forming at around $1.5 \mathrm{MK}$.

In Fig. 2 we show the synthesized $193 \AA$ observation when integrating horizontally through the computational domain. This snapshot reveals a coronal loop hosting $1.5 \mathrm{MK}$ hot plasma. Following the temporal evolution in the movie (available online;

\footnotetext{
${ }^{1}$ Max Planck Institute for Solar System Research, Justus-von-Liebig-Weg 3, 37077 Göttingen, Germany. ${ }^{2}$ Gesellschaft für wissenschaftliche Datenverarbeitung, Am Faßberg 11, 37077 Göttingen, Germany. ${ }^{3}$ Lockheed Martin Solar and Astrophysics Laboratory, Palo Alto, California 94304, USA *e-mail: peter@mps.mpg.de
} 

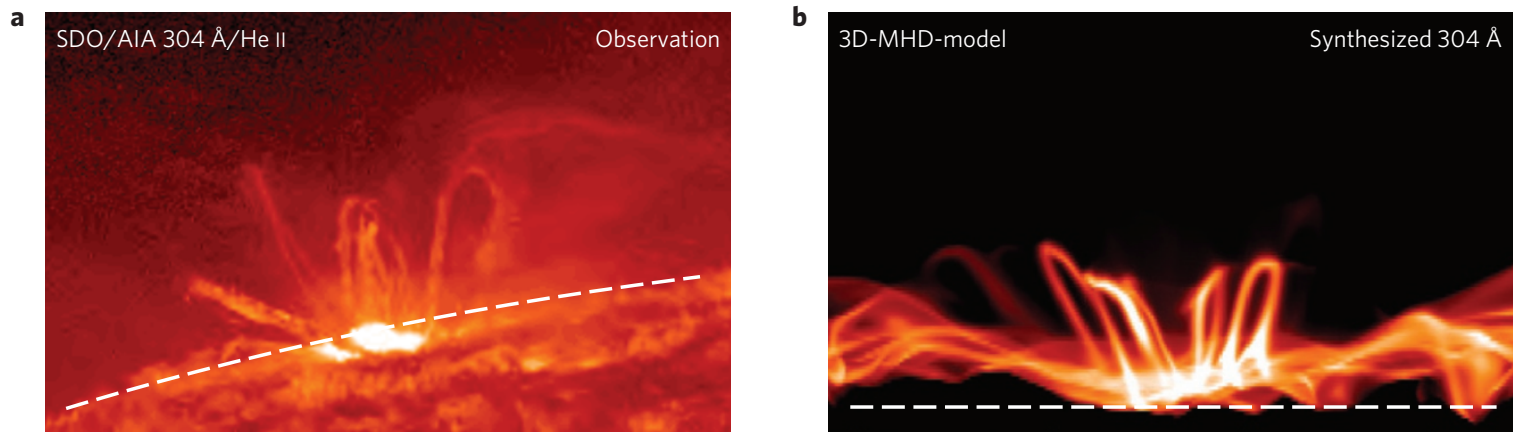

Figure 1 | The upper atmosphere of the Sun seen in light emitted by a hot plasma at approximately 100,000 K. a, An observation from space using the Solar Dynamics Observatory (SDO) taken in the $304 \AA$ band, dominated by emission from singly ionized He. The limb of the Sun is indicated by the dashed line. Coronal loops are mostly seen edge-on, rising some 40,000 km above the limb. $\mathbf{b}$, A numerical simulation as described in this paper. It shows the synthesized emission in the same $304 \AA$ channel, integrated horizontally through the computational box of a numerical experiment. Similar to the real Sun, loops arch above the surface (dashed line).

a

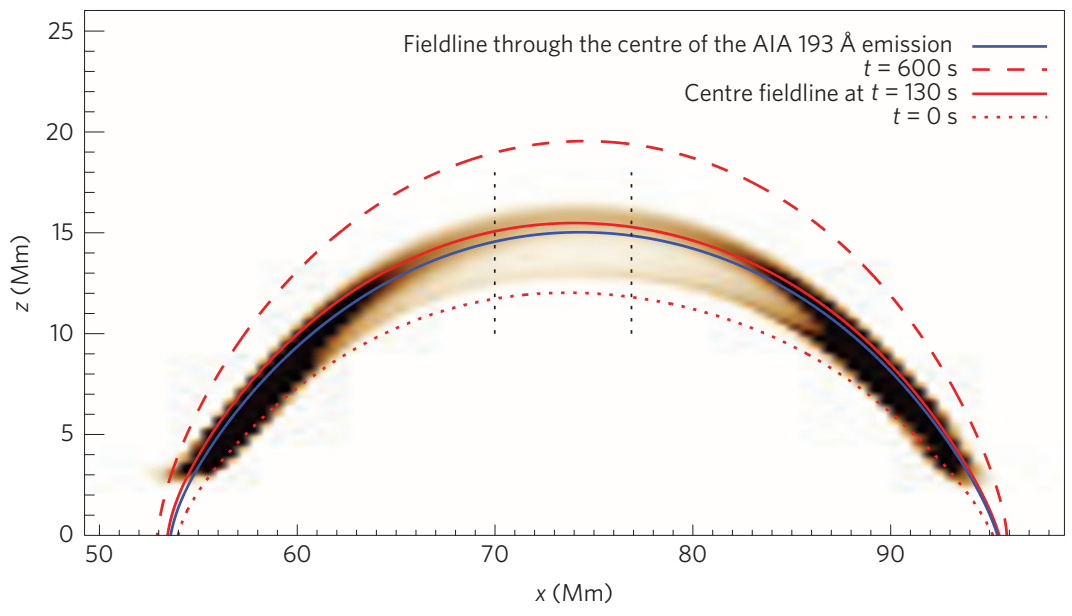

b

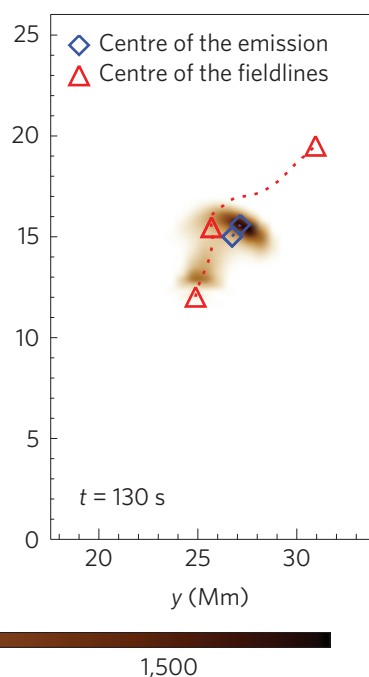

AIA $193 \AA\left(D N\right.$ pixel $\left.^{-1} \mathrm{~s}^{-1}\right)$

Figure $\mathbf{2}$ | Snapshot of the coronal loop in the numerical simulation. The plots show synthesized emission as seen in a wavelength band at $193 \AA$ dominated by Fe XII forming near 1.5 MK. a, View of loop from the side with the emission integrated through the computational box at time $130 \mathrm{~s}$. The emission pattern remains more or less at the same place (see Supplementary Fig. 4 in Supplementary Section 3). In contrast, the field lines expand, here indicated by the same field line shown at three different times $(0 \mathrm{~s}, 130 \mathrm{~s}$ and $600 \mathrm{~s})$. For comparison the blue line shows the field line through the centre of the emission structure at $130 \mathrm{~s}$ (see Supplementary Section 2 for a more precise definition of the red and blue lines). b, A better impression of the 3D structure is obtained by showing the middle part of the loop integrated along the loop (from $x=70 \mathrm{Mm}$ to $77 \mathrm{Mm}$, as indicated by the dotted lines in a). Here the image again shows the $193 \AA$ channel emission, the blue diamond is the centre of the EUV loop and the red triangles the positions of the field line in the $x=74 \mathrm{Mm}$ plane at the same three times as shown in $\mathbf{a}$. These plots cover only part of the computational domain $\left(\approx 150 \times 75 \times 50 \mathrm{Mm}^{3}\right.$ in the $x, y$ and $z$ directions). Supplementary Movie 1 shows the temporal evolution.

further snapshots in Supplementary Fig. 4 in Supplementary Section 3) it is evident that the EUV loop forms, becomes bright and then starts fading over the course of a good fraction of an hour. Most importantly, the EUV loop-that is, the pattern visible in the $193 \AA$ channel-remains at more or less the same place. In particular the EUV loop is not expanding upwards.

This is in contrast to the evolution of the magnetic field. Also in Fig. 2, we overplot one single field line at different times. This field line moves upwards while the active region is emerging. In Fig. 2 (and the movie) we also show the coronal emission in a vertical slab in the middle of the loop (and perpendicular to the loop) to emphasize how differently the pattern of the EUV emission evolves compared to the magnetic structure. There is no mass flow across field lines. We emphasize that at each snapshot the EUV loop is roughly following a field line, but at each time it is a different field line that is aligned with the EUV loop.
To understand this behaviour we investigate the heat input and the resulting temperature, density and emission structure along individual field lines (for details see Supplementary Section 3). Each individual field line undergoes increased Ohmic heating for about $100 \mathrm{~s}$ and through evaporation of gas from the lower atmosphere the density of the loop increases. Because the EUV passbands are sensitive to a limited range of temperatures only, the plasma along each individual expanding field line is brightening up for some $50 \mathrm{~s}$ to $100 \mathrm{~s}$. The field lines get heated in succession, one after the other while moving upwards. This creates a more or less stationary pattern of increased emission, although the structure of the magnetic field is constantly moving upwards. This might be compared to a traffic jam triggered by a construction site on a highway. Here all cars (defining the structure) are moving forward, but the construction site (heating up not the cars but the tempers) and the pile-up of cars (defining the pattern) remain at the same location. 


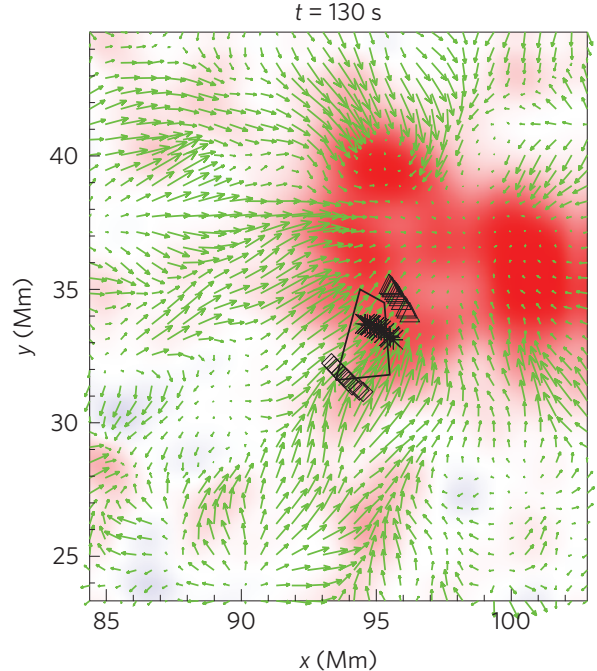

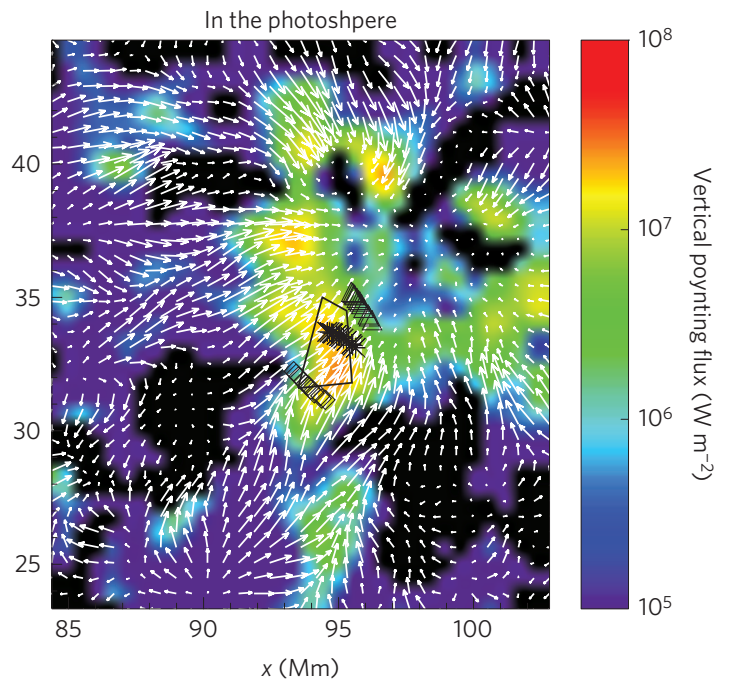

Figure 3 | Evolution at the solar surface while the coronal loop forms. a,b, Vertical magnetic field (a) and vertical component of the Poynting flux (b), both in the photosphere. These snapshots are taken at time $t=130 \mathrm{~s}$. The concentration of magnetic field in $\mathbf{a}$, seen in red, shows the location of one of the two sunspots that form the active region in this simulation. For time $t=-600 \mathrm{~s}$ we indicate a number of positions by the diamonds that are located at the footpoints of field lines that traverse the bright coronal loop later. The asterisks and triangles show the positions of these locations at later times $t=+130 \mathrm{~s}$, and $+1,200 \mathrm{~s}$, when they are carried with the coalescent flow forming the sunspot. The field-of-view covers only a small fraction of the whole computational domain $\left(\approx 150 \times 75 \mathrm{Mm}^{2}\right.$ in the horizontal directions). The temporal evolution over $30 \mathrm{~min}$ from $t=-600 \mathrm{~s}$ to $+1,200 \mathrm{~s}$ is shown in Supplementary Movie 2 .

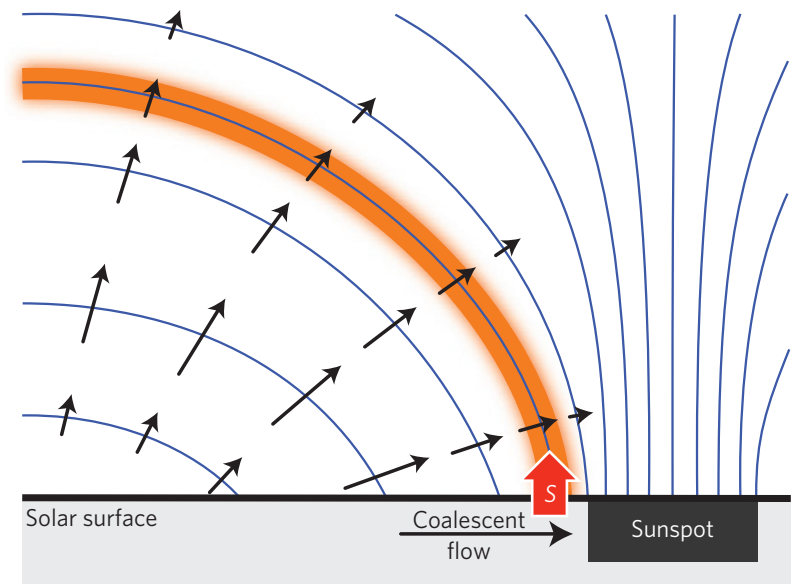

Figure 4 | Cartoon showing the interplay between magnetic field expansion and the EUV loop. A coalescent flow forming the sunspot drags the magnetic field in the photosphere near the solar surface into the sunspot. In response, a hot spot of enhanced upward directed Poynting flux, $S$, forms (red arrow). The expanding field lines (blue) move upwards and to the side. When they transverse the hot spot of Poynting flux, the plasma on that field line gets heated and brightens up. As the field line expands further, it leaves the hot spot and gets darker again. In consequence a bright coronal EUV loop forms (orange) and remains rather stable as the successively heated field lines move through.

The cause for the transient enhancement of the heating of individual field lines is found at their roots. The coalescent flow that forms the sunspot drives magnetic patches towards the strong magnetic field of the sunspot ${ }^{18,24}$. This is illustrated by the arrows in Fig. 3 that indicate the horizontal flows in the photosphere. At the outer edge of the spot there will be a region of enhanced (vertical) Poynting flux - that is, of upward directed flux of magnetic energy. This is similar to the flux-tube-tectonics model ${ }^{20}$, where (horizontal) shuffling of magnetic patches leads to an upward directed flux of magnetic energy, which is then available to heat the coronal plasma. Because each field line is pushed into the spot and thus transverses the region of the enhanced Poynting flux, the heat input into the corona along individual field lines is transient (see detailed discussion in Supplementary Section 3 and movie attached to Supplementary Fig. 2). Thus, EUV loops will show up wherever strong (horizontal) gradients of the magnetic field are present at the footpoints, similar to the tectonics model ${ }^{20}$.

This mechanism is illustrated by the cartoon in Fig. 4. During the emergence of magnetic flux forming a sunspot pair, the field is pushed upwards and to the sides. In sunspots the magnetic field is very strong and convection is suppressed. Thus the flow driving the coalescence of the magnetic field comes to a halt. Whenever a field line crosses the region of enhanced Poynting flux, energy is deposited along that field line and the plasma on it is heated. Consequently this field line becomes visible in EUV for a short time. With successive field lines passing the hot spot of Poynting flux, they all brighten roughly at the same place, creating the illusion of a static emission pattern forming a loop, while the magnetic field is moving. Future work will have to show to what extent this scenario also holds for X-ray emission, which typically forms over a broader range of temperatures than the EUV bands (see Supplementary Section 3).

In our $3 \mathrm{D}$ numerical experiment we find that the temporal evolution of the magnetic field in the corona can be radically different from that of the patterns seen in the coronal emission. This implies that modelling the temporal evolution of EUV loops as $1 \mathrm{D}$ structures following a static field line is a problematic concept in regions where the magnetic field is evolving; namely, whenever the Sun gets dynamic-and interesting. Thus many of the time-dependent 1D loop models that have been used as the workhorse in coronal studies over the past two decades need to be reconsidered. In cases where one can reasonably assume a static magnetic structure confining the plasma, a 1D model might describe the loop sufficiently well. In principle, a model combining several $1 \mathrm{D}$ models could account for changes in loop length ${ }^{25}$ or magnetic connectivity ${ }^{26}$ to mimic the emergence process, but such a multiple-1D-model would suffer from the lack of self-consistency. Only if treated in $(2 \mathrm{D}$ or) $3 \mathrm{D}$ will the photospheric motions that drive the magnetic changes lead to a self-consistent interaction 
of the neighbouring field lines that result in a (variable) spatial pattern of heat input. Still, at any given snapshot the coronal EUV loops in our 3D model outline magnetic field lines. Therefore, EUV observations should provide useful information when implemented into procedures to recover a snapshot of the coronal magnetic field through extrapolation methods ${ }^{27}$. In summary, the magnetic and thermal evolution of the corona should be treated as a coupled system in a single problem - this requires one to have a more holistic view of the magnetic and thermal properties of the corona when addressing the question of the structure, dynamics and heating of the corona.

Received 6 November 2014; accepted 18 March 2015; published online 27 April 2015

\section{References}

1. Peter, H. \& Dwivedi, B. N. Discovery of the Sun's million-degree hot corona. Front. Astron. Space Sci. 1, 2 (2014).

2. Priest, E. Magnetohydrodynamics of the Sun (Cambridge Univ. Press, 2014).

3. Aschwanden, M. J. Physics of the Solar Corona. An Introduction with Problems and Solutions 2nd edn (Springer, 2005).

4. Peter, H. et al. Solar magnetism eXplorer (SolmeX). Exploring the magnetic field in the upper atmosphere of our closest star. Exp. Astron. 33, 271-303 (2012).

5. DeRosa, M. L., Schrijver, C. J., Barnes, G. et al. A critical assessment of nonlinear force-free field modeling of the solar corona for active region 10953. Astrophys. J. 696, 1780-1791 (2009).

6. Aschwanden, M. J., Wülser, J-P., Nitta, N. V. \& Lemen, J. R. First three-dimensional reconstructions of coronal loops with the STEREO A and B spacecraft. I. Geometry. Astrophys. J. 679, 827-842 (2008).

7. Feng, L., Inhester, B., Solanki, S. K. et al. First stereoscopic coronal loop reconstructions from STEREO SECCHI images. Astrophys. J. 671, L205-L208 (2007).

8. Rosner, R., Tucker, W. H. \& Vaiana, G. S. Dynamics of the quiescent solar corona. Astrophys. J. 220, 643-665 (1978).

9. Mariska, J. T., Boris, J. P., Oran, E. S., Young, T. R. \& Doschek, G. A. Solar transition region response to variations in the heating rate. Astrophys. J. 255, 783-796 (1982).

10. Yang, W. H., Sturrock, P. A. \& Antiochos, S. K. Force-free magnetic fields-the magneto-frictional method. Astrophys. J. 309, 383-391 (1986).

11. Cheung, M. C. M. \& DeRosa, M. L. A method for data-driven simulations of evolving solar active regions. Astrophys. J. 757, 147 (2012).

12. Mok, Y., Mikić, Z., Lionello, R. \& Linker, J. A. The formation of coronal loops by thermal instability in three dimensions. Astrophys. J. Lett. 679, L161-L165 (2008).

13. Peter, H. \& Bingert, S. Constant cross section of loops in the solar corona. Astron. Astrophys. 548, A1 (2012).

14. Forbes, T. G. \& Acton, L. W. Reconnection and field line shrinkage in solar flares. Astrophys. J. 459, 330-341 (1996).

15. Gudiksen, B. \& Nordlund, Å. Bulk heating and slender magnetic loops in the solar corona. Astrophys. J. 572, L113-L116 (2002).
16. Bingert, S. \& Peter, H. Intermittent heating in the solar corona employing a 3D MHD model. Astron. Astrophys. 530, A112 (2011).

17. Bourdin, P-A., Bingert, S. \& Peter, H. Observationally driven 3D magnetohydrodynamics model of the solar corona above an active region. Astron. Astrophys. 555, A123 (2013).

18. Rempel, M. \& Cheung, M. C. M. Numerical simulations of active region scale flux emergence: From spot formation to decay. Astrophys. J. 785, 90 (2014).

19. Parker, E. N. Topological dissipation and the small-scale fields in turbulent gases. Astrophys. J. 174, 499-510 (1972).

20. Priest, E. R., Heyvaerts, J. F. \& Title, A. M. A flux-tube tectonics model for solar coronal heating driven by the magnetic carpet. Astrophys. J. 576, 533-551 (2002).

21. Chen, F., Peter, H., Bingert, S. \& Cheung, M. C. M. A model for the formation of the active region corona driven by magnetic flux emergence. Astron. Astrophys. 564, A12 (2014).

22. Peter, H., Gudiksen, B. \& Nordlund, Å. Forward modeling of the corona of the sun and solar-like stars: From a three-dimensional magnetohydrodynamic model to synthetic extreme-ultraviolet spectra. Astrophys. J. 638, 1086-1100 (2006).

23. Lemen, J., Title, A. M., Akin, D. J. et al. The Atmospheric Imaging Assembly (AIA) on the Solar Dynamics Observatory (SDO). Sol. Phys. 275, 14-40 (2012).

24. Cheung, M. C. M., Rempel, M., Title, A. M. \& Schüssler, M. Simulation of the formation of a solar active region. Astrophys. J. 720, 233-244 (2010).

25. Martens, P. C. H. \& Kuin, N. P. M. The thermal evolution of resonantly heated coronal loops. Astron. Astrophys. 123, 216-224 (1983).

26. López Fuentes, M. C. \& Klimchuk, J. A. A simple model for the evolution of multi-stranded coronal loops. Astrophys. J. 719, 591-601 (2010).

27. Malanushenko, A., Schrijver, C. J., DeRosa, M. L. \& Wheatland, M. S. Using coronal loops to reconstruct the magnetic field of an active region before and after a major flare. Astrophys. J. 783, 102 (2014).

\section{Acknowledgements}

We thank R. Cameron for discussions and comments on the manuscript. This work was supported by the International Max-Planck Research School (IMPRS) for Solar System Science at the University of Göttingen. It was was partially funded by the

Max-Planck/Princeton Center for Plasma Physics. The computations were done at GWDG in Göttingen and on SuperMUC in at LRZ in Munich. We acknowledge PRACE for awarding us access to SuperMUC based in Germany at the Leibniz Supercomputing Centre (LRZ). M.C.M.C. acknowledges support from NASA award NNX14AI14G to LMSAL.

\section{Author contributions}

The numerical experiment was designed by H.P. and S.B.; the numerical simulation was conducted by F.C. and S.B.; the analysis of the data was done by F.C., H.P. and S.B.; the boundary conditions were provided and implemented by M.C.M.C. and F.C.; and H.P. and F.C. wrote the text.

\section{Additional information}

Supplementary information is available in the online version of the paper. Reprints and permissions information is available online at www.nature.com/reprints. Correspondence and requests for materials should be addressed to H.P.

\section{Competing financial interests}

The authors declare no competing financial interests. 